\title{
Avaliação in vitro da atividade carrapaticida de Turnera subulata Sm em fêmeas ingurgitadas de Rhipicephalus (Boophilus) microplus
}

\author{
In vitro assessment of the acaridal activity of Turnera subulata Sm in engorged females of \\ Rhipicephalus (Boophilus) microplus \\ Evaluación in vitro de la actividad acaricida de Turnera subulata Sm en teleoginas de Rhipicephalus
} (Boophilus) microplus

\section{Resumo}

A utilização de acaricidas químicos é o principal método de controle das infestações dos carrapatos na bovinocultura e tem sido considerado responsável por comprometer o desenvolvimento da pecuária com grandes perdas econômicas. Turnera subulata Sm. é uma planta popularmente conhecida no Brasil como chanana, rica em bioflavonóides, composto que ocorre em várias plantas medicinais indicadas na medicina popular, sendo testada quanto a suas propriedades acaricidas sobre Rhipicephalus (Boophilus) microplus. O bioensaio in vitro foi baseado no teste de imersão de fêmeas ingurgitadas, no qual o extrato seco de Turnera subulata Sm. diluído seriadamente em concentrações decrescentes de $50 \%$ a $6,38 \%$. Os testes foram realizados com teleóginas molhadas e imediatamente secadas em papel absorvente, indicando que os grupos testados variaram nos seus percentuais de eficiência do produto. Não houve mortalidade das fêmeas adultas e o efeito do extrato associado ao piretróide não apresentou eficácia aceitável, quanto à relação da taxa de eclosão dos ovos obteve-se resultado positivo e houve atividade larvicida, entretanto, as porcentagens apresentadas em todas as concentrações são baixas para o controle de carrapatos. Sugere-se estudos posteriores com o extrato da chanana associados a outros produtos vegetais.

Palavras-chave: Biocarrapatidograma; Chanana; Bovino; Teste de sensibilidade.

\begin{abstract}
The use of chemical acaricides is the main method of controlling infestations of ticks in cattle breeding and has been considered responsible for compromising the development of livestock with great economic losses. Turnera subulata $\mathrm{Sm}$. is a plant popularly known in Brazil as chanana, rich in bioflavonoids, a substance which is found in various medicinal plants indicated in folk medicine and tested for their acaricidal properties on Rhipicephalus (Boophilus) microplus. The in vitro bioassay was based on the immersion test of engorged females, in which the dry extract of Turnera subulata $\mathrm{Sm}$. was diluted at steps indecreasing concentrations from $50 \%$ to $6,38 \%$. This was performed with wet ticks and immediately dried on absorbent paper, indicating that the tested groups varied in their efficiency percentage of the product. There was no mortality of adult females and the effect of the associated extract with pyrethroid did not show plausible effectiveness, regarding the link between egg hatching rate obtained positive result and there was larvicidal activity, however, the percentages presented in all concentrations are low for ticks controlling. It is suggested further studies with chanana extract associated with other plant products.
\end{abstract}

Keywords: Immersion test; Chanana; Cattle; Sensibility test. 


\begin{abstract}
Resumen
El uso de acaricidas químicos es el principal método de control de las infestaciones por garrapatas en el ganado y se ha considerado responsable de comprometer el desarrollo de la ganadería con grandes pérdidas económicas. Turnera subulata Sm. es una planta popularmente conocida en Brasil como chanana, rica en bioflavonoides, compuesto presente en varias plantas medicinales indicadas en la medicina popular, siendo probada por sus propiedades acaricidas sobre Rhipicephalus (Boophilus) microplus. El bioensayo in vitro se basó en la prueba de inmersión de teleoginas en la que el extracto seco de Turnera subulata Sm. se diluyó en serie en concentraciones decrecientes de $50 \%$ a 6,38\%. Las pruebas se realizaron con teleoginas húmedas y se secaron inmediatamente sobre papel absorbente, lo que indica que los grupos evaluados variaron en sus porcentajes de eficiencia del producto. No hubo mortalidad de hembras adultas y el efecto del extracto asociado al piretroide no mostró una eficacia aceptable, ya que para la relación de la tasa de eclosión de los huevos, hubo resultado positivo y hubo actividad larvicida, sin embargo, los porcentajes que se presentan en todas las concentraciones son bajas para el control de garrapatas. Se sugieren más estudios con el extracto de chanana asociado con otros productos vegetales.
\end{abstract}

Palabras clave: Prueba de inmersión; Chanana; Bovino; Prueba de sensibilidad.

\title{
1. Introdução
}

A espécie Rhipicephalus (Boophilus) microplus é um ectoparasita originário da Ásia, que por meio de importação de gados foi introduzida em vários países do mundo. Essa espécie é responsável por ser vetor da conhecida "'tristeza parasitária bovina" e outras doenças que comprometem a saúde do bovino o que pode acarretar perdas econômicas ao produtor comprometendo o desenvolvimento da pecuária (Pereira et al., 2008).

$\mathrm{O}$ uso indiscriminado de acaricida químico tem significância no desenvolvimento de novas espécies de carrapatos resistentes à quimioterapia.

Os danos causados por esses agentes para controle de carrapatos têm incentivado a procura por antiparasitários de origem natural como alternativa de diminuir os danos causados pelos quimioterápicos (Rodrigues et al., 2010).

Duarte (2006) diz que, as plantas são usadas pela população carente devido ao baixo custo e fácil disponibilidade, o interesse por produtos naturais é fonte predominante em países em desenvolvimento como solução para problemas de saúde do animal quanto a preservação do meio ambiente.

O objetivo do estudo foi analisar o efeito da atividade carrapaticida in vitro do extrato seco de Turnera subulata $\mathrm{Sm}$. conhecido popularmente como chanana e, posteriormente, associar o extrato com um acaricida químico piretróide.

\section{Metodologia}

O presente estudo trata-se de uma abordagem quantitativa, baseada em análises laboratoriais da eficácia carrapaticida de Turnera subulata Sm. frente a cepas de carrapatos, distribuídas em grupos expostos a diferentes concentrações do extrato vegetal e sua associação a um composto químico. O tratamento dos dados foi submetido a métodos estatísticos paramétricos e não paramétricos (Turato, 2005).

A coleta de teleóginas e os testes laboratoriais foram desenvolvidos na microrregião de Areia, que se localiza na borda úmida oriental do Planalto da Borborema de acordo com o IBGE -Instituto Brasileiro de Geografia e Estatística, 2006.

A coleta das teleóginas foi realizada em animais que não receberam tratamento com carrapaticida por um período mínimo de 21 dias para que não ocorresse interferência nos resultados dos testes in vitro. As teleóginas com comprimento igual ou superior a $4 \mathrm{~mm}$, foram coletadas manualmente.

Turnera subulata Sm., foi coletada no município de Areia e sua identificação realizada na Universidade Federal da Paraíba no Herbário Jayme Coelho de Morais, número da excicata 21.742. O princípio ativo foi extraído em aparelho de Soxhlet e os solventes foram retirados por rota-vapor durante sete dias na temperatura de $40^{\circ} \mathrm{C}$.

Foi utilizado no estudo um carrapaticida químico, pelo qual a base química é Cipermetrina (piretróide), nota fiscal adquirido em casa de agropecuária, na cidade de Campina Grande-PB. 
O bioensaio in vitro foi baseado no teste de imersão de adultos, pelo qual as fêmeas ingurgitadas foram imersas no extrato seco de Turnera subulata Sm. diluídos seriadamente nas concentrações decrescentes de 50\%, 25\%, 12,5\%, 6,38\%. Posteriormente foi comparada a mortalidade e taxa de oviposição e eclodibilidade de larvas entre as fêmeas do grupo experimental e controle negativo sendo, o mesmo realizado com água destilada para que não houvesse interferência nos resultados (Pereira et al., 2008).

Apenas as teleóginas colhidas nas últimas 48 horas e sem alterações morfológicas ou restos de tegumento no aparelho bucal foram utilizadas no biocarrapaticidograma, no teste foram usados no total de 1.620 teleóginas, segundo metodologia descrita por Wiggers e Stange (2008).

Com auxílio de uma peneira as teleóginas foram lavadas em água corrente, em seguida, secas em papel absorvente e posteriormente foram pesadas em balança analítica, em seguida, foram separadas em triplicatas de grupos homogêneos, contendo 10 teleóginas para cada concentração, com respectivamente o extrato seco de Turnera subulata Sm. a associação do extrato seco com o piretróide, a água destilada como controle negativo. Logo após, as teleóginas de cada grupo permaneceram imersos, durante cinco minutos, em recipientes identificados.

Os testes foram realizados com teleóginas imersas e imediatamente secadas em papel absorvente e com as teleóginas molhadas, para comparar se existia interferência entre ambos os testes, logo após acondicionadas em placas de Petri descartáveis de $100 \mathrm{~mm}$, devidamente identificadas e mantida em recipiente com papel absorvente umidificado para manutenção da temperatura e a umidade relativamente baixa.

Posteriormente, foi realizada a avaliação de mortalidade das teleóginas, pelo qual a massa de ovos de cada grupo foi pesada em balança analítica, armazenada em seringas plásticas descartáveis, previamente adaptadas e identificadas.

Finalmente realizou-se a avaliação da eclodibilidade das larvas e determinado visualmente a estimativa da porcentagem de eclosão dos ovos.

A partir do décimo segundo dia de postura foram devidamente registradas em formulário próprio as principais variáveis da pesquisa: mortalidade das teleóginas, peso das posturas (do $12^{\circ}$ ao $14^{\circ}$ dia de postura), porcentagem de eclodibilidade (50 dias após a pesagem das massas de ovos), eficiência reprodutiva (ER) e eficiência do produto (EP). As variáveis dos biocarrapaticidogramas foram avaliadas, segundo as equações prescritas por (Drummond et al., 1973).

A eficácia do produto foi calculada tendo como base na (E.R.) e (E.P.) para a interpretação dos resultados, considerou-se como eficácia do princípio ativo o resultado igual ou superior a 95\%, conforme a legislação para a comercialização de carrapaticidas (Ministério da Agricultura, 1987).

\section{Resultados e Discussão}

Observou-se que o solvente, água destilada, utilizado como controle negativo, não interferiu nos resultados, uma vez que não foi visualizada a mortalidade das teleóginas ingurgitadas adultas e nem interferência referente à eclosão dos ovos das teleóginas.

Os dados da Figura 1, em relação ao peso das teleóginas e o peso dos ovos, referente a Y e X, apresentam uma estatística significativa entre o peso das teleóginas e o peso dos ovos, que apresentou uma probabilidade de $\mathrm{p}<0.01$, estando dentro da faixa do nível de confiança que foi de $5 \%$. A correlação entre $\mathrm{Y}$ e $\mathrm{X}$ apresentou-se positiva ( $\mathrm{r}=0.68)$ entre $\mathrm{Y}$ e $\mathrm{X}$, onde indica que quando o peso das teleóginas aumenta o peso dos ovos também aumenta. O que explica que em nenhum dos tratamentos houve morte das teleóginas ingurgitadas e consequentemente as teleóginas adultas fizeram a postura dos ovos mostrando que o extrato seco e associado ao acaricida químico não foram eficientes para morte dos carrapatos adultos, no 
entanto, esse estudo pode comprovar a eficácia em relação à redução do tamanho das posturas de Rhipicephalus (Boophilus) microplus.

Figura 1 - Equação ajustada para o modelo linear que descreve a relação entre Peso dos ovos (Y) e Peso das teleóginas (X).

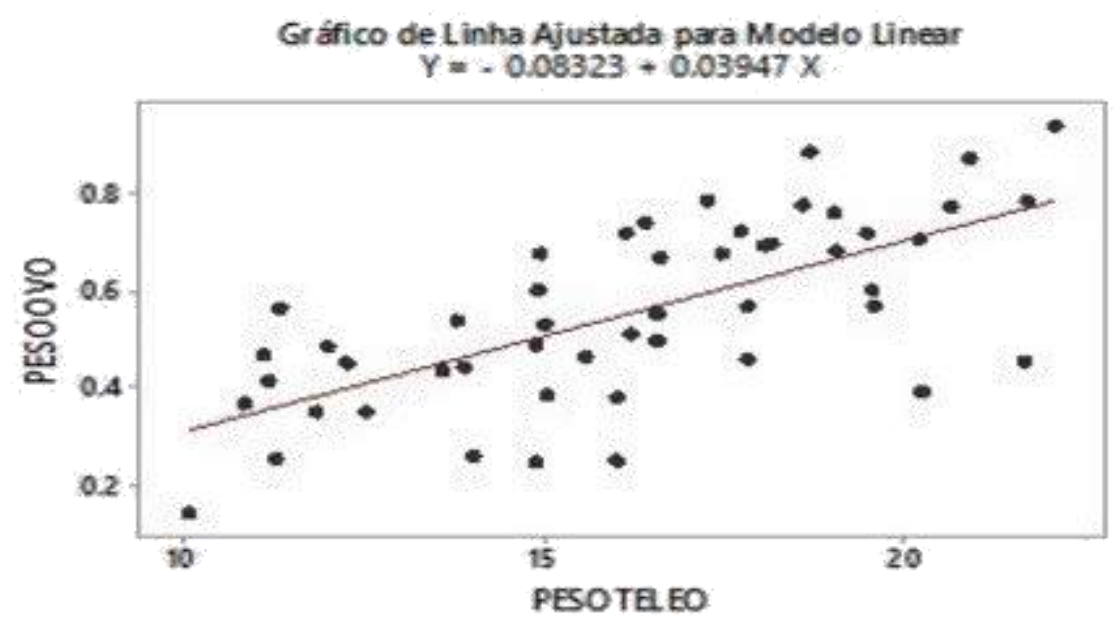

Fonte: Autores.

O tratamento realizado com o extrato seco de Turnera subulata Sm. nas concentrações de 50\%, 25\%, 12,5\% e 6,35\%, pelo qual as teleóginas foram secas com papel absorvente após a imersão do produto, obtiveram os seguintes resultados: $16,66 \%, 5,41 \%, 50,44 \%$ e 12,98\%. A eficácia do extrato seco da chanana que não foi seca após a imersão (molhada) nas concentrações de $50 \%$ e $25 \%$ foram $12,91 \%, 11,35 \%$ e nas concentrações $12,5 \%$ e 6,35\% indicaram valores negativos com eficiência reprodutiva maior que o controle com água destilada, resultando na ineficiência do produto nessas concentrações, possivelmente por ter sido mantidas em ambiente úmido.

Em relação à eficiência do extrato seco da chanana associado ao piretróide, com as teleóginas secadas após a imersão do produto, nas concentrações de 50\%, 25\%, 12,5\% e 6,35\% tiveram os seguintes valores: 8,12\%, 40,97\%, 73,29\% e 10,12\%. Nos extratos da chanana associados com o piretróide, o qual as teleóginas não foram secas após a imersão (molhada) nas concentrações de $50 \%, 25 \%, 12,5 \%$ e $6,35 \%$, as eficiências dos produtos foram respectivamente 70,11\%, 65,21\%, 59,38\% e $71,85 \%$.

Em contrapartida, pesquisas realizadas comprovam que a Turnera subulata Sm. foi uma opção de produto natural com atividade contra parasitas como Trypanossoma cruzi e Leishmania brasiliensis (Santos et al., 2012).

$\mathrm{Na}$ Tabela 1, o extrato seco da chanana na concentração de 12,5\%, no grupo das teleóginas secas após a imersão do produto, teve eficácia de 50,44\%; nessa mesma concentração na Tabela 2, os produtos que tiveram associação do extrato seco da chanana com o piretróide, tanto no grupo das teleóginas secas após a imersão do composto quanto no grupo das teleóginas molhadas, apresentaram eficácia de 73,29\% e 59,38\%, respectivamente. Os resultados mostram que na concentração de $12,5 \%$ o extrato seco da chanana foi mais eficaz, apesar de estarem abaixo do preconizado pelo Ministério da Agricultura, esses resultados revelam a importância de Turnera subulata $\mathrm{Sm}$. como carrapaticida. 
Tabela 1. Tratamento com o extrato seco de Turnera subulata Sm., testadas com teleóginas secas em papel absorvente e molhadas após a imersão do extrato seco. Valores das variáveis avaliadas segundo as equações prescritas por Drummond et al. (1973).

\begin{tabular}{l|c|c|c|c|c}
\hline \multicolumn{1}{c|}{ Tratamentos (\%) } & $\begin{array}{c}\text { Peso da teleógina } \\
(\mathrm{g})\end{array}$ & Massa de ovos (g) & $\begin{array}{c}\text { Eclosão dos } \\
\text { ovos }(\%)\end{array}$ & $\begin{array}{c}\text { Eficiência } \\
\text { reprodutiva }(\%)\end{array}$ & $\begin{array}{c}\text { Eficiência do } \\
\text { produto }(\%)\end{array}$ \\
\hline Controle (água destilada) & 1,8447 & 0,6756 & 97 & $710.502,521$ & - \\
Chanana (seca) 50 & 1,7312 & 0,6250 & 82 & $592.074,861$ & 16,66 \\
Chanana (seca) 25 & 1,4918 & 0,5413 & 92,6 & $671.998,659$ & 5,41 \\
Chanana (seca) 12,5 & 2,1608 & 0,9145 & 41,6 & $352.121,437$ & 50,44 \\
Chanana (seca) 6,35 & 2,2803 & 0,9041 & 79,3 & $628.821,909$ & 12,98 \\
Chanana (molhada) 50 & 1,9232 & 0,7437 & 80 & $618.718,802$ & 12,91 \\
Chanana (molhada) 25 & 1,7958 & 0,6760 & 83,66 & $629.849,204$ & 11,35 \\
Chanana (molhada) 12,5 & 1,7166 & 0,7270 & 94,33 & $798.996,971$ & $-12,45$ \\
Chanana (molhada) 6,35 & 1,6150 & 0,6165 & 97 & $740.563,467$ & $-4,23$ \\
\hline
\end{tabular}

Fonte: Autores.

Tabela 2. Tratamento com o extrato seco da Turnera subulata Sm. e associação com o piretróide, testadas com teleóginas secas em papel absorvente e molhadas após a imersão do extrato seco. Valores das variáveis avaliadas segundo as equações prescritas por Drummond et al. (1973).

\begin{tabular}{|c|c|c|c|c|c|}
\hline Tratamentos (\%) & $\begin{array}{c}\text { Peso da } \\
\text { teleógina }(\mathrm{g})\end{array}$ & $\begin{array}{l}\text { Massa de ovos } \\
\qquad(\mathrm{g})\end{array}$ & $\begin{array}{c}\text { Eclosão dos } \\
\text { ovos }(\%)\end{array}$ & $\begin{array}{c}\text { Eficiência } \\
\text { reprodutiva }(\%)\end{array}$ & $\begin{array}{l}\text { Eficiência do } \\
\text { produto }(\%)\end{array}$ \\
\hline $\begin{array}{l}\text { Controle (água } \\
\text { destilada) }\end{array}$ & 1,8658 & 0,6884 & 97 & $715.880,587$ & - \\
\hline $\begin{array}{l}\text { Chanana (seca) } 50+ \\
\text { piretróide }\end{array}$ & 1,6000 & 0,5720 & 92 & $657.800,000$ & 8,12 \\
\hline $\begin{array}{l}\text { Chanana (seca) } 25+ \\
\text { piretróide }\end{array}$ & 1,3758 & 0,5842 & 50 & $424.625,672$ & 40,97 \\
\hline $\begin{array}{l}\text { Chanana (seca) 12,5 + } \\
\text { piretróide }\end{array}$ & 1,2022 & 0,4597 & 25 & $191.191,150$ & 73,29 \\
\hline $\begin{array}{l}\text { Chanana (seca) 6,35 + } \\
\text { piretróide }\end{array}$ & 1,1817 & 0,4508 & 84,33 & $643.411,424$ & 10,12 \\
\hline $\begin{array}{l}\text { Chanana (molhada) } 50+ \\
\text { piretróide }\end{array}$ & 1,8574 & 0,4482 & 44,33 & $213.941,057$ & 70,11 \\
\hline $\begin{array}{l}\text { Chanana (molhada) } 25+ \\
\text { piretróide }\end{array}$ & 1,7481 & 0,3185 & 68,33 & $248.991,534$ & 65,21 \\
\hline $\begin{array}{l}\text { Chanana (molhada) } 12,5 \\
+ \text { piretróide }\end{array}$ & 1,2139 & 0,2621 & 67,33 & $290.752,006$ & 59,38 \\
\hline $\begin{array}{l}\text { Chanana (molhada) } 6,35 \\
+ \text { piretróide }\end{array}$ & 1,2793 & 0,3222 & 40 & $201.485,187$ & 71,85 \\
\hline
\end{tabular}

Fonte: Autores.

De acordo com a triagem fitoquímica, Turnera subulata $\mathrm{Sm}$. é rica em bioflavonóides presentes como metabólito secundário que são responsáveis pela defesa da planta, esses são utilizados como modelo de pesticida ou como pesticida (Gracioso et al., 2002), podendo dessa forma, justificar a eficiência do produto, pelo qual possibilitou a infusão do principio ativo através das camadas externas, o tegumento coreáceo de Rhipicephalus (Boophilus) microplus.

Mediante a eficácia do extrato de Turnera subulata Sm. sugere-se a combinação do extrato a outro produto que possua princípios ativos carrapaticidas.

Neste contexto, os carrapaticidas naturais possuem certas vantagens em relação aos químicos, pois tendem a ter menor 
toxicidade aos mamíferos, rápida degradação e levam mais tempo para adquirir resistência, tornando os biocarrapaticidas menos agressivos ao meio ambiente e dessa forma gerando menor impacto ambiental (Clemente et al., 2007; Costa-júnior et al., 2016; Juliet et al., 2012; Santos et al., 2018).

Em relação à comparação entre às teleóginas secas após imersão do extrato e molhadas os resultados obtidos no estudo como mostrado nas Tabelas 1 e 2, mostram que os grupos testados variaram amplamente nos seus percentuais de eficiência do produto.

Observou-se que nas concentrações 12,5\% e 6,35\% do extrato seco da Turnera subulata Sm., as que permaneceram molhadas após a imersão do extrato, mostrado na Tabela1, que a eficiência reprodutiva foi maior que o controle, resultando como consequência na eficiência do produto com valor negativo.

Uma explicação plausível seria que os fatores que interferem na incubação e na eclosão dos ovos são ambientes úmidos, pelo fato de Rhipicephalus (Boophilus) microplus apresentarem altas taxas de transpiração e baixas temperaturas críticas de dessecação, portanto os ovos dessa espécie são suscetíveis à umidade (Pereira et al., 2008).

Analisou-se no presente estudo que a baixa umidade favoreceu o desenvolvimento da eclosão dos ovos resultando na ineficiência do extrato, o qual pode ter interferido também na dificuldade de penetração do produto no tegumento das teleóginas.

O efeito interativo do extrato seco da chanana com o piretróide, tanto nas teleóginas secas após a imersão quanto nas que permaneceram molhadas, não apresentou eficiência aceitável conforme a Tabela 2, nas concentrações de 50\%, 25\%, 12,5\% e 6,35\%, segundo preconiza o Ministério da Agricultura, pelo qual, a eficiência legalmente aceitável para que uma base química seja registrada como carrapaticida deve ser no mínimo 95\% sobre uma cepa de $R$. (Boophilus) microplus.

A baixa eficácia do extrato seco combinado com o piretróide pode ser resultado do uso indiscriminado dos carrapaticidas convencionais, por isso, certas estirpes adquiriram resistência intrínseca aos compostos (Andreotti, 2010). Recomenda-se novos estudos que identifiquem o grau de resistência apresentado pelo piretróide frente Rhipicephalus (Boophilus) microplus.

O uso de substâncias químicas acaricidas é o principal método disponível que visa o controle dos carrapatos, cuja principal desvantagem inclui o aumento da resistência do parasita ao princípio ativo (Oliveira et al., 2015; Silva et al, 2008). A classe dos piretróides tem demonstrado maior resistência do que qualquer outra classe acaricida. O local alvo para atividade dos piretroides é o canal de sódio dependente de voltagem e a resistência a essa substância tem sido estudada em várias espécies de artrópodes (Guerreiro et al., 2012; Oliveira et al., 2015).

Os constituintes ativos dos extratos apresentam variações de acordo com: o ciclo circadiano; os fatores fisiológicos, tais como: fotossíntese e crescimento da planta; as variações sazonais, pois a quantidade e a natureza dos constituintes ativos não são constantes durante o ano; os fatores hídricos como, o excesso de chuva (Gobbo-Neto \& Lopes, 2007). Esses fatores podem ter influenciado no resultado do estudo interferindo na qualidade e disponibilidade dos metabólitos secundários tais como, flavonóides, alcalóides, taninos e compostos fenólicos da planta analisada.

A época da coleta de Turnera subulata Sm. foi realizada no mês de junho, período que é marcado pela estação chuvosa na região de Areia, o qual pode resultar na perda de substâncias hidrossolúveis das folhas.

Outros fatores como: condições de coleta, estabilização e estocagem da planta, pode ter grande influência na viabilidade dos princípios ativos da planta; o tipo de solo onde a planta foi colhida pode explicar as discrepâncias nos resultados das pesquisas em diferentes locais, usando a mesma espécie de planta e metodologia (Pinho et al., 2012; Costajúnior et al., 2016).

Conforme o estudo realizado por (Santos et al., 2010) Turnera subulada Sm. foi submetida a teste contra microcrustáceo Artemisia salina, hospedeiro intermediário no ciclo de transmissão da esquistossomose, apesar do extrato não 
apresentar atividade moluscida o extrato foi ativo contra larvas de Artemisia salina, foi observado resultados positivos para glicosídeos cianogênicos, taninos hidrolisáveis, flavonóides, esteróides e alcalóides, os seguintes dados obtidos do bioensaio revelaram que a chanana é uma fonte promissora de substâncias inseticidas, neste contexto, é justificado a eficácia sobre as larvas de Rhipicephalus (Boophilus) microplus.

Pesquisa realizada por Flores et al. (2011) mostraram que o extrato aquoso de uma planta Turneraceae, Turnera difusa, da mesma família da chanana, possui dentre outras atividades, a ação anti-inflamatória e larvicida.

Segundo Antônio e Souza (1998), estudos feitos indicam a eficiência do extrato da Turnera subulata Sm. como excelente agente anti-inflamatório, demonstrando a importância no que diz respeito a inflamação causada pela espoliação do carrapato no couro do bovino podendo ser uma nova alternativa de estudo in vivo.

Após os resultados, o estudo mostrou uma boa eficácia contra as larvas de Rhipicephalus (Boophilus) microplus, dessa forma, é imprescindível ressaltar a necessidade de testes complementares que confirmem a atividade repelente contra as larvas ou efeito larvicida.

\section{Conclusão}

O estudo mostrou efeito positivo, dentro das condições que a pesquisa foi realizada, sobre a eclosão dos ovos de Rhipicephalus (Boophilus) microplus. Sugere-se que Turnera subulata Sm. pode ser uma nova alternativa positiva de combinação com outros produtos vegetais com efeito carrapaticida.

Novos ensaios devem ser desenvolvidos a fim de tentar associar a chanana com outras formulações com extratos vegetais, bem como, avaliar seu potencial sobre estágios imaturos de carrapatos, propondo uma alternativa natural aos produtos químicos amplamente utilizados e que já apresentam resistência.

\section{Referências}

Andreotti, R. (2010). Situação atual da resistência do carrapato-do-boi Rhipicephalus (Boophilus) microplus aos acaricidas no Brasil. Campo Grande, MS: Documentos - Embrapa Gado de Corte, 180.

Antonio, M. A. \& Souza A. R. M. (1998). Oral anti-inflammatory and antiactivities of a hydroalcoholic extract and partitioned fractions of Turnera ulmifolia (Turneraceae). Journal of Ethnopharmacology, 61(1), 215-228. 10.1016/s0378-8741(98)00049-x.

Clemente, M. A., Gomes, F. T., Scotton, A. C. B. S., Goldner, M. S., Reis, E. S. \& Almeida, M. N. (2007). Avaliação do Potencial de Plantas Medicinais no Controle de Boophilus microplus (Acari: Ixodidae). Revista Brasileira de Biociências, 5 (2), 516-518.

Costa-Júnior, L.M., Moleiro, R. J., Alves, P. B., Blank, A. F, Li, A. Y. \& León, A. A. P. (2016). Acaricidal effecacies of Lippia gracilis essential oil its phytochemicals Against organophosphorate-resistant and susceptible strains of Rhipicephalus (Boophilus) microplus. Veterinary Parasitology, $228,60-64$. 10.1016/j.vetpar.2016.05.028.

Drummond, R. O., Ernest, S. E., Trevino, J. L., Gladney, W. J. \& Graham, O. H. (1973). Boophilus annulatus and B. microplus: laboratory tests of insecticides. Journal of Economic Entomology, 66 (1), 130-133. 10.1093/jee/66.1.130.

Duarte, M. C. T. (2006). Atividade Antimicrobiana de Plantas Medicinais e Aromáticas Utilizadas no Brasil. Multi ciência.

Flores, C. A., López, C. C., Montejo, F. J. \& Reys-Leys-Leyva, J. (2011). Atividade antiproliferativa de Turnera difusa Willd. Revista Latinoamericana de Quimica. 38.

Gobbo-Neto, L. \& Lopes, N. P. (2007). Plantas Medicinais: Fatores de Influência do Conteúdo de Metabólitos Secundários. Química Nova, 30 (2), 374-381. $10.1590 / \mathrm{S} 0100-40422007000200026$.

Gracioso, J. S., Vilelas, W., Hiruma, L. C. A. \& Souza, B. A. R. (2002). Effects of tea from Turnera ulmifolia L on mouse gastric mucosa support the Turneraceae as a new source of antiulcerogenic drugs. Biological and Pharmaceutical Bulletin, 25(1), 487 - 491. 10.1248/bpb.25.487

Guerreiro, F. D., Lovis, L. \& Martins, J. R. (2012). Acaricide resistance mechanisms in Rhipicephalus (Boophilus) microplus. Revista Brasileira de Parasitologia Veterinária, 21 (1), 1- 6. 1 0.1590/S1984-29612012000100002.

Juliet, S., Ravindranb, R., Ramankuttyb, S. A., Gopalanb, A. K. K., Naira, S. N., Kavillimakkilb, A. K., Bandyopadhayayc, A., Rawtd, A. K. S. \& Ghoshe, S. (2012). Jatropha curcas (Linn) leaf extract - a possible alternative for population control of Rhipicephalus (Boophilus) annulatus. Asian Pacific Journal of Tropical Disesase, 2 (3), 225-229. 10.1016/S2222-1808(12)60051-6. 
Research, Society and Development, v. 10, n. 9, e31910918198, 2021

(CC BY 4.0) | ISSN 2525-3409 | DOI: http://dx.doi.org/10.33448/rsd-v10i9.18198

Ministério da Agricultura (1987). Normas para registros de parasiticidas de uso pecuário no Brasil.

Oliveira, M. C. S., Brito, L. G., Barbieri, F. S., Gonçalves, T. C., Tanaka, E., Giglioti, R., Bilhassi, T. B., Santana, R. C. M., Néo, T. A., Chagas, A. C. S. \& Rabelo, M. D. (2015). Resistência aos pesticidas piretróides em populações de Rhipicephalus microplus e aos piretróides e organofosforados em Haematobia irritans colhidas em rebanhos de corte no Estado de São Paulo. São Paulo, SP: Boletim de Pesquisa e Desenvolvimento - Embrapa Pecuária Sudeste, 38.

Pereira, M. C., Labruna, M. B., Szabó, M. P. J. \& Klafke, G. M. (2008). Rhipicephalus (Boophilus) microplus Biologia, Controle e Resistência. MedVet.

Pinho, L., Souza, P. N. S., Sobrinho, E. M., Almeida, A. C. \& Martins, E. R. (2012). Antimicrobial activity of hidroalcoholic extracts from rosemary, Pepper tree, barbatimão and erva baleeira leaves and from pequipeel meal. Ciência rural, 42 (2), 03-20.

Rodrigues, S., Rodrigues, M. \& Cruz, C. (2010). The acaricide effect of Ethanolic Extracts of Some Plants on Tics Rhipicephalus (Boophilus) microplus. La Revista MVZ Córdoba, 15 (3), 2175- 2184

Santos, N. C., Dias, C. N., Moraes, D. F. C., Vilanova, C. M., Gonçalves, J. R. S., Souza, N. S. \& Rosa, I. G. (2010). Toxicidade e avaliação de atividade moluscicida de folhas de Turnera ulmifolia L. Revista Brasileira de Biociências, 8 (4), 324-329.

Santos, K. K., Matias, E. F. F., Sobral-Souza, C. E., Tintino, S. R., Morais-Braga, M. F. B., Guedes, G. M. M., Rolón, M., Veja, C., Rojas, A., Costa, J. G. M., Menezes, I. R. A. \& Coutinho, H. D. M. (2012). Evaluation of the anti-Trypanosoma and anti-Leishmania activity of Mentharvensis and Turnera ulmifolia L. Boletín Latinoamericano y del Caribe de Plantas Medicinales y Aromáticas, 2 (11), 147-153.

Santos, B. M., Bruno Filho, F. B. \& Mendes, M. M. (2018). Avaliação da ação de extratos vegetais com potencial atividade carrapaticida. PUBVET, 12 (8) p.1-7. 10.31533/pubvet.v12n8a157.1-7.

Silva, F. F., Soares, M. C. S. C., Alves, L. C., Lima, M. M., Silva, L. V. A., Faustino, M. A. G. \& Silva Júnior, F. F. (2008). Comparative evaluation of the efficacy of phythotherapeutics and chemical products against tick in the control of the Boophilus microplus (Canestrini, 1887) through engorged female bioassay. Medicina Veterinária, 2 (3), 1-8.

Turato, E. R. (2005). Métodos qualitativos e quantitativos na área da saúde: definições, diferenças e seus objetos de pesquisa. Revista de Saúde Pública, 39(3), 507-514.

Wiggers, I. \& Stange, C. E. B. (2008). Manual de instrução para coleta, identificação e herborização de material botânico. 